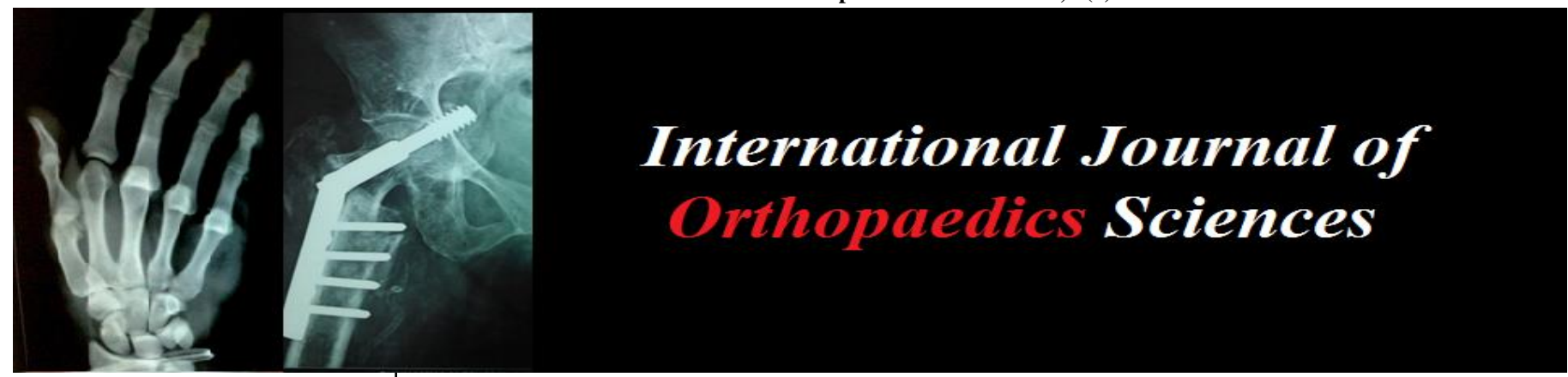

ISSN: $2395-1958$

IJOS 2018; 4(3): 194-199

(C) 2018 IJOS

www.orthopaper.com

Received: 06-05-2018

Accepted: 07-06-2018

Dr. Sujai S

MVJ Medical College \& Research Hospital, Dandupalya, Kolathur Post, Kolar Highway, Hoskote, Bangalore, Karnataka, India

\section{Dr. Akshay TM}

MVJ Medical College \& Research Hospital, Dandupalya, Kolathur Post, Kolar Highway, Hoskote,

Bangalore, Karnataka, India

Dr. Momin Bin Latheef

MVJ Medical College \& Research Hospital, Dandupalya, Kolathur Post, Kolar Highway, Hoskote,

Bangalore, Karnataka, India

Dr. Nataraj G

MVJ Medical College \& Research Hospital, Dandupalya, Kolathur Post, Kolar Highway, Hoskote,

Bangalore, Karnataka, India

Dr. MK Siddalinga Swamy MVJ Medical College \& Research Hospital, Dandupalya, Kolathur Post, Kolar Highway, Hoskote, Bangalore, Karnataka, India
Correspondence

Dr. Sujai S

MVJ Medical College \& Research Hospital, Dandupalya, Kolathur Post, Kolar Highway, Hoskote,

Bangalore, Karnataka, India

\section{Management of osteoporotic extracapsular fractures of the proximal femur with proximal femur locking plate: A prospective study}

\author{
Dr. Sujai S, Dr. Akshay TM, Dr. Momin Bin Latheef, Dr. Nataraj G and \\ Dr. MK Siddalinga Swamy
}

DOI: $\underline{\text { https://doi.org/10.22271/ortho.2018.v4.i3d.36 }}$

\section{Abstract}

Introduction: Proximal femur fractures are a cause for significant morbidity \& mortality in the elderly. These fractures need to be fixed surgically so as to help rehabilitate the patient at the earliest. Extra capsular fractures (Intertrochanteric fractures \& Sub trochanteric fractures) when occur in osteoporotic bone are usually comminuted in nature. Choice of implant is of prime importance as the risk of implant failure is very high in such patients. This study was undertaken to see the functional outcome in management of osteoporotic proximal femoral bone fractures treated by open reduction \& internal fixation with Proximal Femoral Locking Plate.

Materials \& Methods: In this prospective study conducted at MVJMC\&RH, 40 elderly patients with Extra capsular fractures of the proximal femur (IT \& ST) were treated with surgical fixation using PFLP from October 2014 to march 2017.

Results: In the present study of 40 cases with an average age of patient is 65years with Male to Female ratio of 42.5\%: 57.5\%; 26 Intertrochanteric fractures \& 14 Sub trochanteric fractures the outcome was found to be $25 \%$ (10) excellent, $32.5 \%$ (13) good, $32.5 \%$ (13) fair, $10 \%$ (4) poor result with Harris hip scoring system. 4 complications include 1 infection; 2 delayed union \& 1 case of purely implant related complication like plate bending due to early mobilization \& weight bearing.

Conclusion: In view of the results obtained from our study which are comparable with the results obtained from similar studies; despite few unfavourable results and complications, Proximal FLP is a good option for treatment of proximal femur fractures with severe comminution and osteoporosis.

Keywords: Extra capsular hip fracture, intertrochanteric \& sub trochanteric femur fracture, unstable proximal femur fracture, proximal femoral locking plate (PFLP)

\section{Introduction}

With the advent of modern medicine, the average lifespan of Indians has increased from $48 \mathrm{yrs}$ in 1970 to $70 y r s$ presently. With the increase in lifespan, the incidence of osteoporotic fractures in the elderly population is on the rise. These fractures result from a trivial fall.

Proximal femur fractures are a cause for significant morbidity \& mortality in the elderly. These fractures needs to be fixed surgically so as to help rehabilitate the patient at the earliest. Early mobilization \& rehabilitation have a significant impact in decreasing the morbidity \& mortality in such patients.

Extracapsular fractures of the proximal femur may be of the following types:

- Inter trochanteric fractures

- Sub trochanteric fractures.

These fractures when occur in osteoporotic bone are usually comminuted in nature. The modality of fixation of such fractures is complicated by features such as comminution \& osteoporosis. Choice of implant is of prime importance as the risk of implant failure is very high in such patients.

Dynamic Hip Screw (DHS) has been the gold standard treatment of stable IT fractures. However, newer modalities of fracture fixation have been devised for unstable IT \& sub trochanteric fractures. These comprise of IM implants like Proximal Femoral Nail (PFN) \& plate systems such as Proximal Femoral Locking Plate (PFLP). 
PFLP provides the surgeon with flexibility to achieve plate to bone apposition, adequate alignment of fracture, stabilize the fracture reduction satisfactorily without extensive periosteal stripping. Biomechanical studies have shown PFLP to be stronger than other modalities. They have the mechanical advantage of 3 dimensional \& angularly stable fixations.

Cut out of proximal screws through osteoporotic head is a common complication in DHS \& PFN fixation. But the incidence of the same is less with PFLP. It also provides a greater degree of variability \& also fulfils the role of a fixed angle device at the same time.

In view of the above consideration, we have chosen PFLP as the implant of choice for Extracapsular osteoporotic fractures of the proximal femur in our study.

\section{Materials \& Methods}

This is a prospective study conducted at MVJMC\&RH from October 2014 to march 2017.

40 Elderly patients with Extracapsular fractures of proximal femur (IT \& ST) were treated with surgical fixation using PFLP.

The patients were reviewed periodically both clinically \& radiologically for a minimum of 6 months.

Criteria like hip movements following surgery, complications, time required for union were evaluated.

\section{Inclusion Criteria}

- $\quad$ Patients above 50yrs of age with extracapsular fractures (IT \& ST) of proximal femur.

\section{Exclusion Criteria}

- Open injuries.

- Pathological fractures.

- Multiple/severe coexisting Medical co-morbidities.

\section{Protocol}

1. Patients above 50yrs of age presenting with Extracapsular proximal femoral fractures (ST / IT) were admitted to our hospital.

2. A thorough history was elicited from the patient/attenders with regards of the nature of the injury (mechanical fall or due to any other causes).

3. The affected limb was placed in a Thomas splint/ skin traction to maintain length \& alignment.

4. Taking consent for further treatment /surgery.

5. All preoperative investigations were carried out Blood CBC, ESR, FBS/PPBS/ HBA1C/ RBS, RFT, LFT, Serum Electrolytes, HIV, HBsAg, BT, CT, APTT, INR, Urine Routine, Blood grouping/ Rh typing + Cross matching.

Special Investigations: - (if required)

CT Brain- to R/O intracranial injuries/ Pathologic causes. ECHO- for preoperative cardiac evaluation.

6. X-rays-

a) Pelvis with b/l hip- AP view

b) Lateral view of affected side

c) Traction \& internal rotation views

d) Chest- PA view for preoperative check-up.

7. After obtaining clearance for surgery from medical \& anaesthetic teams patient was posted for Open Reduction \& Proximal Femoral Locking Compression Plate fixation.

8. The procedure carried out under Spinal Anaesthesia / Epidural Anaesthesia or combined.

9. The patient was placed on a radiolucent fracture table with the affected limb in longitudinal traction.
10. Under C-arm guidance fracture alignment was achieved with closed reduction \& rotational alignment as required.

11. The surgical site $\&$ the entire lower limb was scrubbed with betadine surgical scrub. Parts painted \& draped appropriately.

12. Incision of appropriate length placed over the lateral aspect of hip \& thigh extending proximally from the tip of Greater Trochanter into the shaft distally.

13. Fascia lata incised \& vastus lateralis split accordingly.

14. Fracture site \& entire lateral aspect of femur \& trochanter visualised.

15. Major / Large comminuted fracture fragment aligned. Periosteal stripping avoided. Muscle interposition removed.

16. Plate placed on lateral aspect of proximal femur \& anchored temporarily with $\mathrm{K}$-wires through the holes provided for the same.

17. 3 proximal screws of appropriate length with pre fixed angles of- $\left[95^{\circ}, 120^{\circ}, 135^{\circ}\right]$ proximal to distal are introduced after drilling with provided locking sleeves with the help of $\mathrm{C}$-arm. Atmost care taken to make sure the second screw $\left(120^{\circ}\right)$ is placed centrally in both AP \& lateral planes as the first \& third screw go anteriorly \& posteriorly in the neck.

18. The remaining distal locking screws are then placed on to the distal shaft with due consideration given to working length of the construct. Minimum of 5 screws used distally.

19. Final alignment \& fixation checked with $\mathrm{C}$-arm in both AP \& Lateral planes.

20. Thorough wound wash given, drain placed, wound closed in layers \& sterile dressing applied.

21. Patient received appropriate IV antibiotics, analgesics, DVT prophylaxis \& Antigastritic measures.

22. All medical co-morbidities were treated / managed simultaneously \& patient received appropriate medication for respective co-morbidities.

23. Post op wound inspection \& drain removal on day $3^{\text {rd }}$. Post op $\mathrm{x}$-rays of hip with femur- AP/Lateral view obtained.

24. Appropriate bed care, position change, bed side chest physiotherapy, limb physiotherapy \& knee ROM exercises carried on Day 3 or earlier as tolerated by patient.

25. Post op wound dressings done on Day $7^{\text {th }}, 11^{\text {th }} \& 14^{\text {th }}$. Suture removal done on Day $14^{\text {th }} / 15^{\text {th }}$ according to the wound condition.

26. Patient mobilised Non Weight Bearing / Partial Weight Bearing (toe touch only) as tolerated by patient with walker support.

27. DVT prophylaxis continued for minimum 6 weeks post op.

28. Patient followed up at 4 weeks interval until fracture union.

29. During follow up criterias such as- Hip \& Knee ROM; Clinical \& radiological evaluation done to access fracture union, functional outcome assessed as per Harris Hip Score \& graded accordingly.

30. All complications with regards to our fixation noted \& results tabulated accordingly.

\section{Results}

The following observations were made from data collected during study.

Total cases: $-40$ 
Age range:

$-50-80 y r s$

Average age:

$-65 y r s$

- 17 / 23

Side:

- Right side - 24

Left side

$-16$

Inter Trochanteric

$-26$

-14 .

Mechanism of Injury: - Self fall.

Table 1: Sex Distribution

\begin{tabular}{|c|c|c|}
\hline Sex & No of Cases & Percentage \\
\hline Male & 17 & 42.5 \\
\hline Female & 23 & 57.5 \\
\hline
\end{tabular}

Table 2: Type of Fracture

\begin{tabular}{|c|c|c|}
\hline Type of Fracture & No of Cases & Percentage \\
\hline Intertrochanteric & 26 & 65 \\
\hline Subtrochanteric & 14 & 35 \\
\hline
\end{tabular}

Table 3: Side Affected

\begin{tabular}{|c|c|c|}
\hline Side Affected & No of Cases & Percentage \\
\hline Right & 24 & 60 \\
\hline Left & 16 & 40 \\
\hline
\end{tabular}

Table 4: Age Distribution

\begin{tabular}{|c|c|c|}
\hline Age Group (in years) & No of cases & Percentage \\
\hline $51-60$ & 7 & 17.5 \\
\hline $61-70$ & 14 & 35 \\
\hline $71-78$ & 19 & 47.5 \\
\hline
\end{tabular}

Table 5: Complications

\begin{tabular}{|c|c|c|}
\hline Complications & No of Cases & Percentage \\
\hline Implant Failure & 1 & 2.5 \\
\hline Delayed Union & 2 & 5 \\
\hline Infection & 1 & 2.5 \\
\hline Nil & 36 & 90 \\
\hline
\end{tabular}

Table 6: Harris Hip Score

\begin{tabular}{|c|c|c|}
\hline Results & No of Cases & Percentage \\
\hline Excellent & 10 & 25 \\
\hline Good & 13 & 32.5 \\
\hline Fair & 13 & 32.5 \\
\hline Poor & 4 & 10 \\
\hline
\end{tabular}

Table 7: Bone Union

\begin{tabular}{|c|c|c|}
\hline Fracture Union (In Weeks) & No of Cases & Percentage \\
\hline$\leq 16 \mathrm{WKS}$ & 3 & 7.5 \\
\hline $16-20 \mathrm{WKS}$ & 13 & 32.5 \\
\hline $20-24 \mathrm{WKS}$ & 20 & 50 \\
\hline$\geq 24 \mathrm{WKS}$ & 4 & 10 \\
\hline
\end{tabular}

We followed up all the patients until union of fractures ranged from 16-24 weeks.

The average time for union of fracture was 20 weeks ranged from 16-24 weeks.

We had total 4 complications include 1 infection; 2 delayed union \& 1 case of purely implant related complication plate bending.

\section{Discussion}

Initial treatment of the proximal femur fractures in the $1800 \mathrm{~s}$ was mainly non-surgical. The major advances in the treatment of femoral fractures were first seen in 1870 when Hugh Owen
Thomas developed the Thomas splint, and advocated immobilization with prolonged bedrest ${ }^{[1]}$. Surgical management of the hip fractures started gaining interest when the first Bone plate was used in 1886 when Hanmann devised his retrievable bone plate. Lambotte, Lane (1914) Sherman (1912) and Townsend \& Gilfillan (1943) played an important role in development of the modern principles of osteosynthesis ${ }^{[2]}$. The real modern era of internal fixation of hip fractures began with the invention of the triflange nail by Smith-Peterson in $1925^{[3]}$. The invention of sliding compression with a cannulated system of drilling and insertion was invented by Godoy-Moreira and is the precursor of this class of implants in 1938. The desire to increase stability of unstable fracture patterns with valgus osteotomies was popularized by Dimon and Houston, Sarmiento, Harrington in the 1960s \& 1970s ${ }^{[4]}$. Cephalomedullary implants are devices inserted with a closed technique and fluoroscopic control with variable femoral length geometry and enhanced proximal geometry to permit fixation with nails or screws into the femoral head. The Grosse-Kempf gamma nail and the Russel-Taylor reconstruction nail were the start of two new classes of intra-medullary devices designed for the hip region. Locked and hybrid compression plates have been applied recently for unstable fractures with only preliminary results so far. Stable fractures of the proximal femur can be easily treated with osteosynthesis with conventional implants with predictable results. However, management of unstable fractures is a challenge for the surgeon because of difficulty in obtaining anatomical reduction. Several plate designs have been developed over the last decades for the treatment of unstable fractures ${ }^{[5]}$.

Most Intertrochanteric fractures occur in people with poor bone quality, about half of the intertrochanteric fractures are comminuted and unstable. In these fractures, excessive medialization of the shaft and subsequent loss of contact between the fragments can lead to fixation failure. Even after the fracture ultimately unites, limb shortening and decreased length of the abductor lever arm adversely affect hip function. Therefore, unstable hip fractures should be differentiated from their stable counterparts with regard to treatment plan and prognosis. The locking compression plate was introduced in the 21 st century as a new implant that allows angular stable plating for the treatment of complex comminuted and osteoporotic fractures. The Locking Compression Plate (LCP) has the option of using the dynamic compression hole or the threaded locking hole or both. This combination provides the flexibility of cortex screw or locking screw fixation ${ }^{[6-10]}$.

Biomechanical studies have shown LCPs to achieve stronger and stiffer fixation than other angularly stable implants ${ }^{[6]}$. More recently, locking plates especially designed for the proximal femur, PF-LCP have become available especially for the management of complex trochanteric fractures ${ }^{[11]}$.

The PF-LCP was reported to be the strongest construct for vertically orientated femoral neck fractures among 4 different fixation techniques [12]. The PF-LCP with the 'kickstand' screw was reported to have similar biomechanical properties as the $95^{\circ}$-angled blade plate ${ }^{[13]}$. One study reported that the PF-LCP fixation achieved a union rate of $100 \%$ in 110 patients with per trochanteric femoral fractures at the one-year follow-up ${ }^{[14]}$.

Nonetheless, screw breakage after PF-LCP fixation is a complication in per trochanteric fractures with missing posteromedial corners, which leads to high axial bending forces around the fracture site with eventual Varus collapse of the fracture and screw breakage ${ }^{[15-16]}$. Other reported 
complications include loss of fixation with and without screw breakage and plate breakage; the failure rate was independent of the surgeon's experience ${ }^{[17]}$.

\section{Age of Incidence}

The average age incidence in our study was 65 years. This is in contrast to higher age group as reported by western literatures. The average life expectancy of an Indian is 10 years less than western standards. Malnutrition and osteoporosis go hand in hand.

Our study results are comparable with. Guo-Chun Zha ${ }^{[19]}$ and Wang Y

\begin{tabular}{|c|c|}
\hline Author & Average Age \\
\hline Biao Zhong ${ }^{[20]}$ & 68 \\
\hline Boydd and Griffin $^{[18]}$ & 69.7 \\
\hline Guo-Chun Zha $^{[19]}$ & 75 \\
\hline Present study & 65 \\
\hline
\end{tabular}

\section{Sex incidence}

In the present study male: female was 42.5: 57.5. There was a female sex preponderance seen in our study which is similar to other studies of the following authors.

\begin{tabular}{|c|c|c|c|}
\hline Author & Year & Female & Male \\
\hline \multirow{2}{*}{ Hunter \& Krajbich $^{[24]}$} & 1978 & $62(70.4 \%)$ & $26(26.6 \%)$ \\
\cline { 2 - 4 } & 1982 & $185(64.4 \%)$ & $102(35.6 \%)$ \\
\hline \multirow{2}{*}{ GS Kulkarni ${ }^{[25]}$} & 1984 & $76(55 \%)$ & $64(45 \%)$ \\
\cline { 2 - 4 } & 1998 & $117(60.6 \%)$ & $76(39.6 \%)$ \\
\hline Present study & 2017 & $23(57.5 \%)$ & $17(42.5 \%)$ \\
\hline
\end{tabular}

Indian females are mainly confined to household activities and are more prone to slip \& fall while climbing up / down stairs or while going to bathroom.

\section{Mode of injury}

All extracapsular fracture in this study resulting from slip and fall.

The percentage of slip \& fall cases in studies done by Gupta $\mathrm{R}$ C [26] (1974) \& Ganz et al. ${ }^{[25]}$ (1979) were $79.4 \%$ \& $70 \%$ respectively.

\section{Side involved}

In the present study out of 40 cases Right hip fractures were seen in 24 cases and Left side were 16. In studies conducted by Wade P A and R C Gupta ${ }^{[26]}$ right sided fractures were more common, whereas in studies made by Kenzor et al. and Cleveland et al. left side fracture were common.

\section{Distribution according to co-morbid status}

In our study anaemia was most common co morbid condition. Hypertension, diabetes \& obesity were also associated. Details given below

\begin{tabular}{|c|c|c|}
\hline Co morbidities & No of cases & Percentage \\
\hline Anaemia & 32 & 80 \\
\hline Hypertension & 14 & 35 \\
\hline Diabetes Mellitus & 8 & 20 \\
\hline Coronary Artery Disease & 1 & 2.5 \\
\hline Renal Disease & 1 & 2.5 \\
\hline HTN \& DM & 8 & 20 \\
\hline
\end{tabular}

\section{Duration of hospital stay}

Duration of hospital stay in our study was found to be ranging from 11 to 28 days; mean duration being 16.6 \pm 4.06 days.

\section{Postoperative complications}

In the present study pain around the hip joint was the most important post op complaint. We had total 4 complications include 1 superficial infection; 2 delayed union \& 1 case of purely implant related complication like, bending of implant.
Implant failure in our study we found were because of early weight bearing and mobilization.

\section{Evaluation of hip}

Pain: Pain is an important criterion for evaluation of hip fractures which could be due to implant failure, joint penetration, infection, due to avascular necrosis etc. Most of cases had no pain after fracture union. $4 \%$ had mild-moderate degree of pain not affecting activities which subsided by medication.

During the evaluation of cases at the end of $24^{\text {th }}$ week with Harris hip scoring system; the outcome was found to be $25 \%$ (10) excellent, $32.5 \%$ (13) good results and 32.5\% (13) had fair \& $10 \%$ (4) poor result.

\section{Bone union time}

\begin{tabular}{|c|c|}
\hline Author & Average Bone union time (wks) \\
\hline Biao Zhong $^{[20]}$ & $16 \mathrm{wks}$ \\
\hline Hodel S $^{[21]}$ & $16 \mathrm{wks}$ \\
\hline Kovalak E $^{[22]}$ & $22 \mathrm{wks}$ \\
\hline Shah MD $^{[23]}$ & $20 \mathrm{wks}$ \\
\hline Present Study $^{2}$ & $20 \mathrm{wks}$ \\
\hline
\end{tabular}

\section{Conclusion}

The terms of successful outcome include a good understanding of fracture biomechanics, proper patient selection, good preoperative planning, accurate instrumentation and exactly performed osteosynthesis. The overall results were GOOD in this study group. Proximal Femoral Locking Plate is a good method for proximal femur fractures in the elderly patients especially for severely communited fracture and with osteoporosis. The results obtained from our study are comparable with the results obtained from similar studies conducted which we have discussed above. Hence we conclude that the Proximal Femoral Locking Plate, despite few unfavourable results and complications, is a satisfactory method of managing proximal femur fractures with severe comminution and osteoporosis. 
Pre-operative and post - operative X-rays

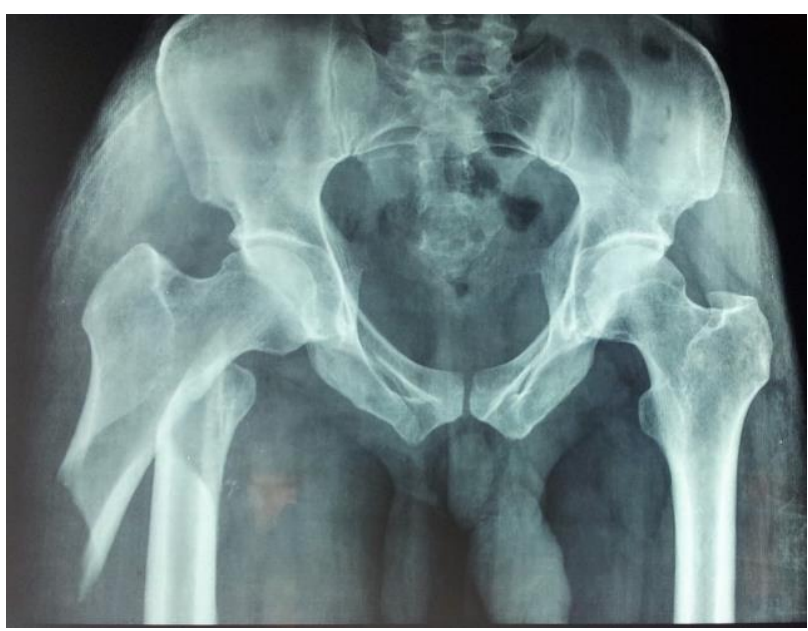

1.

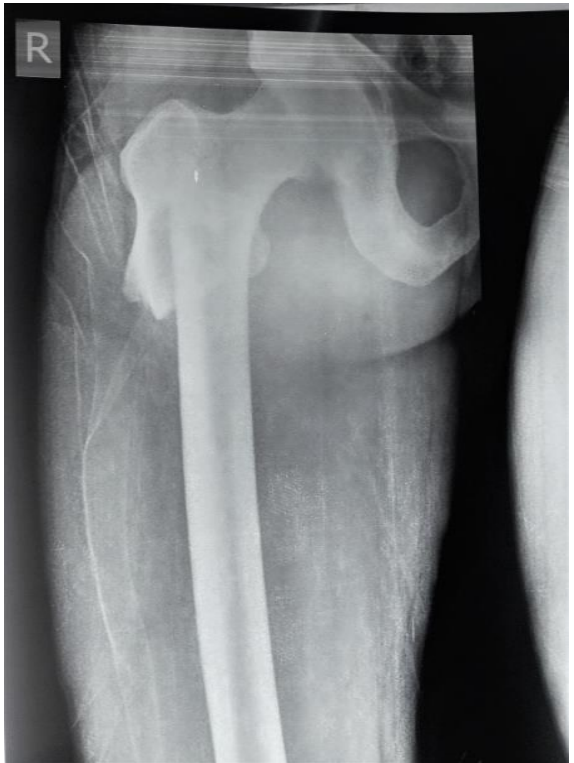

3.

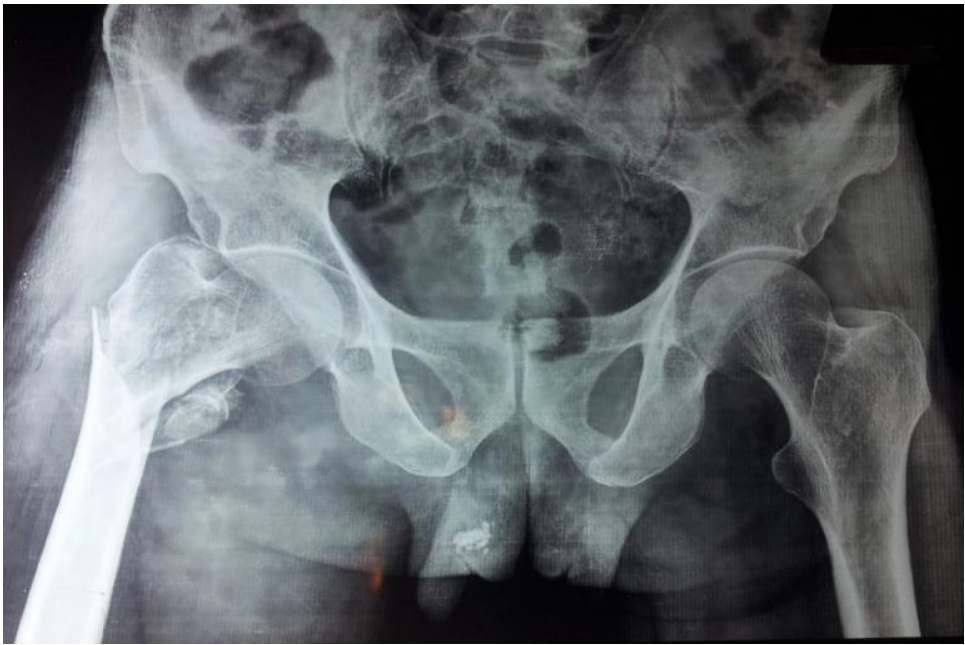

5 .

\section{References}

1. Hugh Owen Thomas. Quoted by Rockwood CA, Green DP. Fracture in Adults, 4th ed. 1972e; 2:73.

2. Townsend Kenneth, Gilfillan Charles. A new type of bone plate and screws. Surg Gynecol Obstet. 1943;

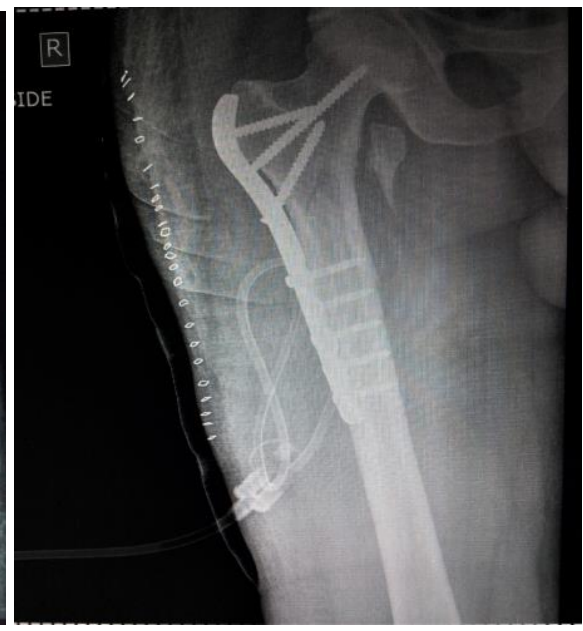

2.

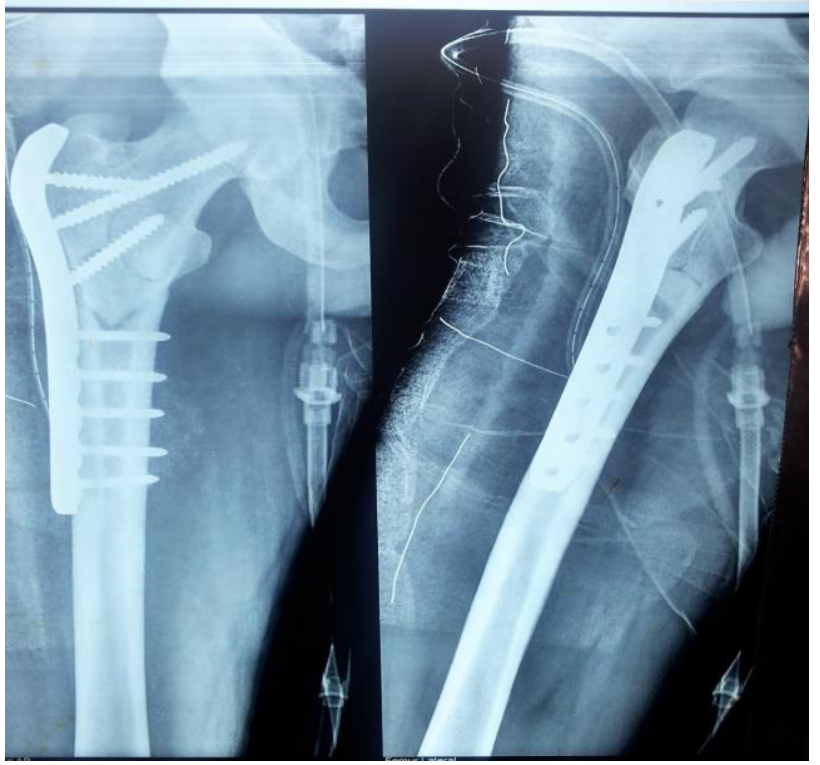

4.

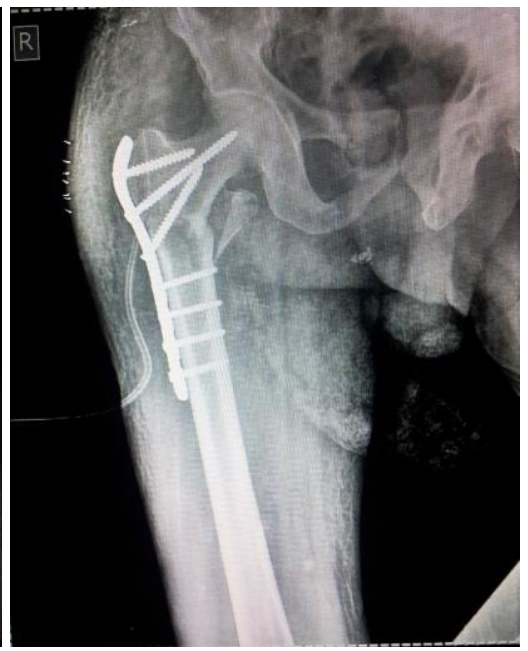

6.

77:595e597.

3. Smith Peterson M. Treatment of neck of femur by internal fixation. Surg Gynecol Obstet. 1937; 64:287.

4. Dimon JHJC. Unstable intertrochanteric fractures of the hip. J Bone Joint Surg Am. 1967; 49(3):440e450. 
5. Medoff RM. A new device for the fixation of unstable pertrochanteric fractures of the hip. J Bone Joint Surg Am. 1991; 73(8):1192e1199.

6. Koval KJ, Zuckerman JD. Hip fractures: II. Evaluation and treatment of intertrochanteric fractures. J Am Acad Orthop Surg. 1994; 2:150e156.

7. Wieser K, Babst R. Fixation failure of the LCP proximal femoral plate $4.5 / 5.0$ in patients with missing posteromedial support in unstable per-, inter-, and subtrochanteric fractures of the proximal femur. Arch Orthop Trauma Surg. 2010; 130:1281e1287.

8. Haidukewych GJ, Israel TA, Berry DJ. Reverse obliquity fractures of the intertrochanteric region of the femur. $\mathbf{J}$ Bone Joint Surg Am. 2001; 83:643e650.

9. Sadowski C, Lubbeke A, Saudan M. Treatment of reverse oblique and transverse intertrochanteric fractures with use of an intramedullary nail or a 95 screw-plate: a prospective, randomized study. J Bone Joint Surg Am. 2002; 84:372e381.

10. Archdeacon M, Ford KR, Wyrick J. A prospective functional outcome and motion analysis evaluation of the hip abductors after femur fracture and antegrade nailing. J Orthop Trauma. 2008; 22:3e9.

11. Forte ML, Virnig BA, Kane RL. Geographic variation in device use for intertrochanteric hip fractures. J Bone Joint Surg Am. 2008; 90:691e699.

12. Yoo MC, Cho YJ, Kim KI, Khairuddin M, Chun YS. Treatment of unstable peritrochanteric femoral fractures using a 95 degrees angled blade plate. J Orthop Trauma. 2005; 19:687-92.

13. Crist BD, Khalafi AK, Hazelwood SJ, Lee MA. A biomechanical comparison of locked plate fixation with percutaneous insertion capability versus the angled blade plate in a subtrochanteric fracture gap model. J Orthop Trauma. 2009; 23:622-7.

14. Zha GC, Chen ZL, Qi XB, Sun JY. Treatment of pertrochanteric fractures with a proximal femur locking compression plate. Injury. 2011; 42:1294-9.

15. Evaluation of proximal femoral locking plate in unstable extracapsular proximal femoral fractures: Surgical technique \& mid-term follow up results. Journal of Clinical Orthopaedics and Trauma. 2014; 5(3)137.

16. Wieser K, Babst R. Fixation failure of the LCP proximal femoral plate $4.5 / 5.0$ in patients with missing posteromedial support in unstable per-, inter-, and subtrochanteric fractures of the proximal femur. Arch Orthop Trauma Surg. 2010; 130:1281-7.

17. Glassner PJ, Tejwani NC. Failure of proximal femoral locking compression plate: a case series. J Orthop Trauma. 2011; 25:76-83.

18. Boyd HB, Griffin LL. Classification and Treatment of Trochanteric Fractures; Arch. Surg. 1949; 58:853-866.

19. Guo-Chun Zha, Ze-Lin Chen, Xiao-Bo Qi, Jun-Ying Sun. Orthopaedic department, The First Affiliated Hospital of Soochow University, 188 Shizi 118 Street, Suzhou, Jiangsu 215006, PR China; Orthopaedic Department, The People's Hospital of Zongyang, Zongyang, Anhui 246702, PR China.

20. Zhongetal B. Orthopaedics \& Traumatology: Surgery \& Research. 2014; 100:663-668.

21. Hodel S, Beeres FJP, Babst R, Link BC. Eur J Orthop Surg Traumatol. 2017; 27(8):1117-1124.

22. Kovalak E, Ermutlu C, Atay T, Başal Ö. J Clin Orthop Trauma. 2017; 8(3):209-214.

23. Shah MD, Kapoor CS, Soni RJ, Patwa JJ, Golwala PP. J
Clin Orthop Trauma. 2017; 8(4):308-312.

24. Davis TR, Sher JL, Checketts RG, Porter BB. Intertrochanteric Fractures of the Femur: A Prospective Study Comparing the Use of the Kuntscher - Y Nail and Sliding Hip Screw; Injury. 1988; 19(6):421 6.

25. Kulkarni GS. Treatment of Trochanteric Fractures of the Hip by Modified Richard's Compressing and Collapsing Screw; Indian Journal of Orthopaedics. 1984; 18(1):3034.

26. Gupta RC. Conservative Treatment of Intertrochanteric Fractures of the Femur; Indian Journal of Orthopaedics. 1974; 36(6):229. 